\title{
A mini review of functional magnetic resonance imaging
}

\author{
Xue $\operatorname{Han}^{1}$ and Tianliang $\mathrm{Li}^{2}$ \\ ${ }^{1}$ School of Mechanical and Electronic Engineering, Wuhan University of Technology, P.R.China \\ ${ }^{2}$ Department of Biomedical Engineering, National University of Singapore, Singapore
}

\begin{abstract}
fMRI, which captures images reflected the hemodynamic changes during the neural activity, shows great potential to uncover the neurophysiological relationship with the brain function and the neuropathological mechanism involved in brain lesions. In this article, an introduction of functional magnetic resonance imaging (fMRI) is presented on its imaging principle, instrumentation and methods, data analysis and current application.
\end{abstract}

\section{Introduction}

The major development in the study of brain function and lesions were made possible by the development of functional brain mapping techniques. The mechanism of functional brain mapping techniques is based on either electromagnetic activity or hemodynamic changes. Both electromagnetic and hemodynamic physiology signals are related to neural activity and neuro-vascular coupling.

Functional magnetic resonance imaging (fMRI) is one of the commonly used brain mapping techniques, which generates images that reflects which brain areas are activated (and how) during external stimuli or performance of different tasks. fMRI shows superiority over other brain mapping techniques: it is noninvasive and has excellent spatial resolution on the order of several millimeters, which makes it greatly developed over the past two decades to become the dominant technique in the study of brain science and lesion diagnosis [1].

The aim of this paper is to review the imaging principle, instrumentation, experiment design and data analysis of fMRI as well as its fundamental and clinical application.

\section{The physics and physiological principle of fMRI}

The physics of fMRI involves the interaction between the biological tissue and electromagnetic fields [1], while its physiological principle relies on the BOLD (blood oxygenation level dependent) effect.

Human brain needs a constant oxygen supply in order to maintain its functional and structural integrity. A special mechanism, which has to provide oxygen during both resting state and neural activity, is necessary to ensure the correct oxygenation level. The strict coupling existing between 'activation', local oxygen consumption and increased regional blood flow constitutes the basis of the so called BOLD effect $[2,3]$.

When the participants undergo a MR scanning, the nuclei or protons in the brain region of interest aligns with the direction of magnetic field of the MR scanner. A radio-frequency (RF) sequence is then applied, causing the nuclei to alter their magnetization alignment. When the RF field is removed, the nuclei go back to their original state. By applying another gradient magnetic field that vary linearly over space, different nuclei to be spatially localized and specific slices to be imaged can be obtained [4]. A paramagnetic substance in the blood, known as deoxygenated hemoglobin (deoxy-Hb), can cause the nuclei to lose magnetization faster via the $\mathrm{T}_{2}^{*}$ decay [5,6], which is called transverse relaxation time. Thus, a pulse sequences sensitive to $\mathrm{T}_{2}{ }^{*}$ show more MR signal where blood is highly oxygenated and less where it is not [7]. Usually the inhaled oxygen is more than the oxygen consumed in burning glucose, which causes a net decrease in deoxy- $\mathrm{Hb}$ in the brain region's blood vessels, as a result, its decrease leads to a less interference with the magnetization and an improved MR signal [8-10].

\section{Data acquisition and data analysis}

Generally, a fMRI image is obtained through four steps.

\section{Experiment design}

The determining factor whether a fMRI detecting is successful or not is how to design the experiment paradigms. According to the current literature reported, there are two kinds of stimuli paradigms. One is called blocked design, which implements the stimuli periodically, like, $\mathrm{ABABAB}$..., $\mathrm{A}$ is the resting-state, $\mathrm{B}$ is the stimulus state, and $\mathrm{AB}$ is one cycle [11]. It measures average neural activity across a block of closely spaced, successive stimuli of the same type [12]. The other one is named event-related design, which records the neural activity on the condition of a single event, for example, at the sight of an unfamiliar picture or memorizing some words, and plays an important role in the research of cognitive neuroscience.

\section{Data acquisition}

Most fMRI studies use standard MR scanners to detect changes in brain blood flow during the performance of cognitive tasks or paradigms designed to elicit neural activation.

Generally, the intensity of MR signal is proportional to the strength of magnetic field. However, the ultrahigh magnetic field is harmful to

Correspondence to: Xue Han (PhD), School of Mechanical and Electronic Engineering, Wuhan University of Technology, Wuhan, P.R.China, Tel: 0086-13659890327, Fax:0086-27-87651793, E-mail: hanxue@whut.edu.cn / 20724308@qq.com

Received: February 05, 2018; Accepted: February 22, 2018; Published: February 25,2018 
human being and always to be applied in animal research. Clinically, the most used magnetic field is $1.5 \mathrm{~T}$.

Besides the strength of magnetic field, different fMRI images can be obtained by selecting the scanning sequences. The commonly used are spin echo (SE) sequence and echo planar imaging (EPI) sequence, which have an ideal time resolution and less motion artifacts.

Additionally, the setting of scanning parameters, such as the repetition time (TE), the echo time (RE), the flip angle, the slice oblique, the slice thickness, the slice gap and the matrix size should also be designed according to different experimental needs.

\section{Preprocessing}

The goal of fMRI data analysis is to explore correlations between brain activation and a task the participant performs during scanning. Before the data analysis, the raw images should be preprocessed.

The aim of preprocessing is to improve the signal-to-noise ratio (SNR), which mainly includes the following procedures:timing correction, motion correction, normalization, temporal filtering and spatial smoothing [13].

The MR scanner acquires different slices within a single brain volume at different times, and hence the slices represent brain activity at different time points. a timing correction is applied to bring all slices to the same time point reference.

Since the fMRI signal is so weak that a slight head movement or rotation may produce artifacts in it. Motion correction is applied to produce the smoothest time course for all voxels.

In order to make a comparison of fMRI scanning results among different participants, a normolization is implemented to adjust all the results to align to a standard template. The commonly used brain atlas are the Talairach one and the Montreal Neurological Institute (MNI) one.

Temporal filtering is the removal of frequencies of no interest from the raw signal, while spatial smoothing is aimed to average the intensities of nearby voxels to produce a smooth spatial map of intensity change across the brain or region of interest.

All the procedures in preprocessing on the raw data, a fourdimensional (4D) magnitude time-series, can be implemented through SPM software, thus, a spatially aligned and normalized dataset for each participant is obtained for the following data analysis.

\section{Data analysis: Brain area localization or brain functional connectivity}

Until now, there is no standard data analysis method or result evaluation criterion. The classical statistic analysis, such as correlation analysis, t-test and non-parametric statistics has been originally used as a tool for fMRI data analysis, which is also named as the hypothesisdriven approach. Recently, some methods of pattern recognition, like, clustering technique, principal component analysis (PCA) and independent component analysis (ICA) have been applied increasingly, which is regarded as the data-driven approach [14-16]. Data processing and analysis techniques play an important role in the research of brain science based on fMRI, which is also a research hotspot in the field of fMRI.

\section{Application}

In the last 20 years, extensive applications of fMRI in the research of brain science have been reported, which could be summarized into two topics $[17,18]$ : one area of study is brain functional connectivity, which aims to localize brain areas linked to basic functions, such as vision [19], auditory [20,21], language [22,23], moving [24], cognition[25-27], etc. The other one is the neurophysiological mechanism of severe brain diseases. Clinically, fMRI is carried out to anatomically examine the brain and provides insights into the physiopathogenesis of brain injury or diseases, such as epilepsy [28,29], stroke [30], Alzheimer's disease [31], Parkinson's disease [32], schizophrenia [33], conduct disorder $[34,35]$, etc. It is regarded as an predictive supplementary method for diagnosis, treatment and prognosis.

\section{Conclusion}

As a promising brain mapping technique, some meaningful progress and valuable research results have been obtained in the study of brain science based on fMRI. However, fMRI has a limited time resolution, is highly sensitive to motion artifact and can only provide an indirect measurement of neural activity. An increasing number of studies have focused on the multimodal brain mapping techniques for the research in neuroscience or physiopathology [36-39]. The other well-known functional imaging techniques include electroencephalography (EEG), magnetoencephalography (MEG), positron emission tomography (PET) and functional near infrared spectroscopy (fNIRS). It is believed that these techniques in combination could effectively improve the precision and accuracy and will grow more and more important in future study.

\section{References}

1. Huttel SA, Song AW, McCarthy G (2009) Functional Magnetic Resonance Imaging (2 edition). Massachusettes: Sinauer, ISBN: 978-0-87893-286-3.

2. Ogawa S, Menon RS, Tank DW, Kim SG, Merkle H, et al. (1993) Functional brain mapping by blood oxygenation level-dependent contrast magnetic resonance imaging. A comparison of signal characteristics with a biophysical model. Biophys $J$ 64: 803812. [Crossref]

3. Molinari F, Liboni W, Grippi G, Negri E (2006) Relationship between oxygen supply and cerebral blood flow assessed by transcranial Doppler and near-infrared spectroscopy in healthy subjects during breath-holding. J NeuroEng Rehabil 3: 1-13. [Crossref]

4. Weishaupt D, Kochli VD, Marincek B, Kim EE (2006) How does MRI work? An introduction to the physics and function of magnetic resonance imaging. Springer 48: 1910.

5. Logothetis NK (2008) What we can do and what we cannot do with fMRI. Nature 453: 869-878. [Crossref]

6. Ogawa S, Lee TM, Nayak AS, Glynn P (1990) Oxygenation-sensitive contrast in magnetic resonance image of rodent brain at high magnetic fields. Magn Reson Med 14: 68-78. [Crossref]

7. Logothetis NK, Pauls J, Augath M, Trinath T, Oeltermann A (2001) Neurophysiologica investigation of the basis of the fMRI signal. Nature 412: 150-157. [Crossref]

8. Weishaupt D, Victor D, Koechli, Marincek B. How does MRI work? An introduction to the physics and function of magnetic resonance imaging. Springer Science \& Business Media. ISBN: 9783662078051.

9. Poustchi-Amin M, Mirowitz SA, Brown JJ, McKinstry RC, Li T (2001) Principles and applications of echo-planar imaging: a review for the general radiologist. Radiographics 21: 767-779. [Crossref]

10. Feinberg DA, Moeller S, Smith SM, Auerbach E, Ramanna S, et al. (2010) Multiplexed echo planar imaging for sub-second whole brain FMRI and fast diffusion imaging. PLoS One 5: e15710. [Crossref]

11. Nie SD, Wang H, Li W, Wu J (2005) Brain functional magnetic resonance imaging and value of its processing and analysis techniques in nerve science. Chinese Journal of Clinical Rehabilitation 9: 162-165.

12. Macpherson H, Formica M, Harris E, Daly RM (2017) Brain functional alternation in Type 2 Diabetes - A systematic review of fMRI studies. Front Neuroendocrinol 47: 34-46. [Crossref]

13. Mitra PP, Pesaran B (1999) Analysis of dynamic brain imaging data. Biophys $J 76$ 691-708. [Crossref] 
14. Baumgartner R, Ryner L, Richter W, Summers R, Jarmasz M, et al. (2000) Comparison of two exploratory data analysis methods for fMRI: fuzzy clustering vs. Principal component analysis. Magn Reson Imaging 18: 89-94. [Crossref]

15. McKeown MJ, Makeig S, Brown GG, Jung TP, Kindermann SS, et al. (1998) Analysis of fMRI data by blind separation into independent spatial components. Hum Brain Mapp 6: 160-188. [Crossref]

16. Levin DN, Uftring SJ (2001) Detecting brain activation in fMRI data without prior knowledge of mental event timing. Neuroimage 13: 153-160. [Crossref]

17. Turner R, Howseman A, Rees GE, Josephs O, Friston K (1998) Functional magnetic resonance imaging of the human brain: data acquisition and analysis. Exp Brain Res 123: 5-12. [Crossref]

18. Folkers P, Boesiger P (2001) A compact 3.0T MR system for routine application, Medicamundi 45: 2-12.

19. Weng XG, Wang HN, Zhang ZQ (2009) Study on the resting-state visual network in patients with anisometropic amblyopia based on fMRI data. Acta Biophys Sin 25: $447-452$.

20. Cheung SW, Nagarajan SS, Bedenbaugh PH, Schreiner CE, Wang X, et al. (2001) Auditory cortical neuron response differences under isoflurane versus pentobarbital anesthesia. Hear Res 156: 115-127. [Crossref]

21. Cheung MM, Lau C, Zhou IY, Chan KC, Cheng JS, et al. (2012) BOLD fMRI investigation of the rat auditory pathway and tonotopic organization. Neuroimage 60 : 1205-1211. [Crossref]

22. Karunanayaka P, Schmithorst VJ, Vannest J, Szaflarski JP, Plante E, et al. (2010) A group independent component analysis of covert verb generation in children: a functional magnetic resonance imaging study. NeuroImage 51: 472-487. [Crossref]

23. Yasuda CL, Centeno M, Vollmar C, Stretton J, Symms M, et al. (2013) The effect of topiramate on cognitive fMRI. Epilepsy Res 105: 250-255. [Crossref]

24. Khushu S, Kumaran SS, Tripathi RP, Gupta A, Jain PC, et al. (2001) Functional magnetic resonance imaging of the primary motor cortex in humans: response to increased functional demanels. $J$ Biosci 26: 205-215.

25. Hutchinson JB, Uncapher MR, Wagner AD (2015) Increased functional connectivity between dorsal posterior parietal and ventral occipitotemporal cortex during uncertain memory decisions. Neurobiol Learn Mem 117: 71-83. [Crossref]

26. Francesco Scalici, C Galtahirone, Carlesimo GA (2017) The contribution of different prefrontal cortex regions to recollection and familiarity: a review of fMRI data. Neurosci Biobehav Rev 83: 240-251. [Crossref]
27. Sidhu MK, Stretton J, Winston GP, Symms M, Thompson PJ, et al. (2015) Memory fMRI predicts verbal memory decline after anterior temporal lobe resection. Neurology 84: 1512-1519. [Crossref]

28. Tang Y, Xia W, Yu X, Zhou B, Wu X, et al. (2016) Altered cerebral activity associated with topiramate and its withdrawal in patients with epilepsy with language impairment: an fMRI study using the verb generation task. Epilepsy Behav 59: 98-104. [Crossref]

29. Xiao FL, An DM, Zhou D (2017) Functional MRI-based connectivity analysis: A promising tool for the investigation of the pathophysiology and comorbidity of epilepsy. Seizure 44: 37-41. [Crossref]

30. Canazza A, Minati L, Boffano C, Parati E, Binks S (2014) Experimental models of brain ischemia: a review of techniques, magnetic resonance imaging, and investigational cell-based therapies. Front Neurol 5: 19. [Crossref]

31. Wang L, Zang Y, He Y, Liang M, Zhang X, et al. (2006) Changes in hippocampal connectivity in the early stages of Alzheimer's disease: evidence from resting state fMRI. Neuroimage 31: 496-504. [Crossref]

32. Luo C, Song W, Chen Q, Zheng Z, Chen K, et al. (2014) Reduced functional connectivity in early-stage drug-naive Parkinson's disease: a resting-state fMRI study. Neurobiol Aging 35: 431-441. [Crossref]

33. Manoach DS, Gollub RL, Benson ES, Searl MM, Goff DC, et al. (2000) Schizophrenic subjects show aberrant fMRI activation of dorsolateral prefrontal cortex and basal ganglia during working memory performance. Biolo Psychiatry 48: 99-109. [Crossref]

34. Blair RJ, Leibenluft E, Pine DS (2014) Conduct disorder and callous-unemotional traits in youth. $N$ Engl J Med 372: 784. [Crossref]

35. Blair RJ, Veroude K, Buitelaar JK (2016) Neuro-cognitive system dysfunction and symptom sets: A review of fMRI studies in youth with conduct problems. Neurosci Biobehav Rev. [Crossref]

36. Herweg NA, Apitz T, Leicht G, Mulert C, Fuentemilla L, et al. (2016) Theta-alpha oscillations bind the hippocampus, prefontal cortex, and striatum during recollection: evidence from simultaneous EEG-fMRI. J Neruosci 36: 3579-3587. [Crossref]

37. He B, Liu Z (2008) Multimodal functional neuroimaging: Integrating functional MRI and EEG/MEG. IEEE Rev Biomed Eng 1: 23-40. [Crossref]

38. Ou W, Nummenmaa A, Hamalainen M, Golland P (2009) Multimodal functional imaging using fMRI-informed regional EEG/MEG source estimation. Inf Process Med Imaging 21: 88-100. [Crossref]

39. Minati L, Grisoli M, Franceschetti S, Epifani F, Granvillano A, et al. (2012) Neural signature of economic parameters during decision-making: A functional MRI (fMRI), Electroencephalograph (EEG) and Autonomic Monitoring study. Brain Topogr 25: 7396. [Crossref]

Copyright: (C2018 Han X. This is an open-access article distributed under the terms of the Creative Commons Attribution License, which permits unrestricted use, distribution, and reproduction in any medium, provided the original author and source are credited. 